WellBeing International

WBI Studies Repository

2019

\title{
The Potential of Organ on Chip Technology for Replacing Animal Testing
}

Malcolm Wilkinson

Kirkstall Ltd.

Follow this and additional works at: https://www.wellbeingintlstudiesrepository.org/humctri

Part of the Bioethics and Medical Ethics Commons, Clinical Trials Commons, and the Laboratory and Basic Science Research Commons

\section{Recommended Citation}

Wilkinson, M. (2019). The Potential of Organ on Chip Technology for Replacing Animal Testing. In Animal Experimentation: Working Towards a Paradigm Change (pp. 639-653). Brill.

This material is brought to you for free and open access by WellBeing International. It has been accepted for inclusion by an authorized administrator of the WBI Studies Repository. For more information, please contact wbisr-info@wellbeingintl.org.

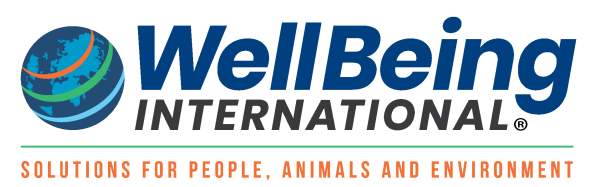




\title{
The Potential of Organ on Chip Technology for Replacing Animal Testing
}

\author{
Malcolm Wilkinson \\ Chief Executive Officer, Kirkstall Ltd., Rotherham, United Kingdom \\ malcolm.wilkinson@kirkstall.com
}

\section{What Is Organ on a Chip Technology?}

The term organ on a chip is used to describe the latest stage of development of in vitro cell culture technology. Figure 26.1 shows its steady development since the 196os. Each step forward has improved our ability to model human-clinical response to new drugs or therapies and has enabled safety risk assessment of existing cosmetics, personal care products, or other chemicals in the environment. Scientific evidence that the predictive power of in vitro tests is superior to the use of animals will trigger a major shift in the way that medical research, in many areas, is carried out. In this emerging field, some researchers also refer to organ on a chip as a microphysiological system. As yet, there are few agreed upon standards or definitions for the latest developments.

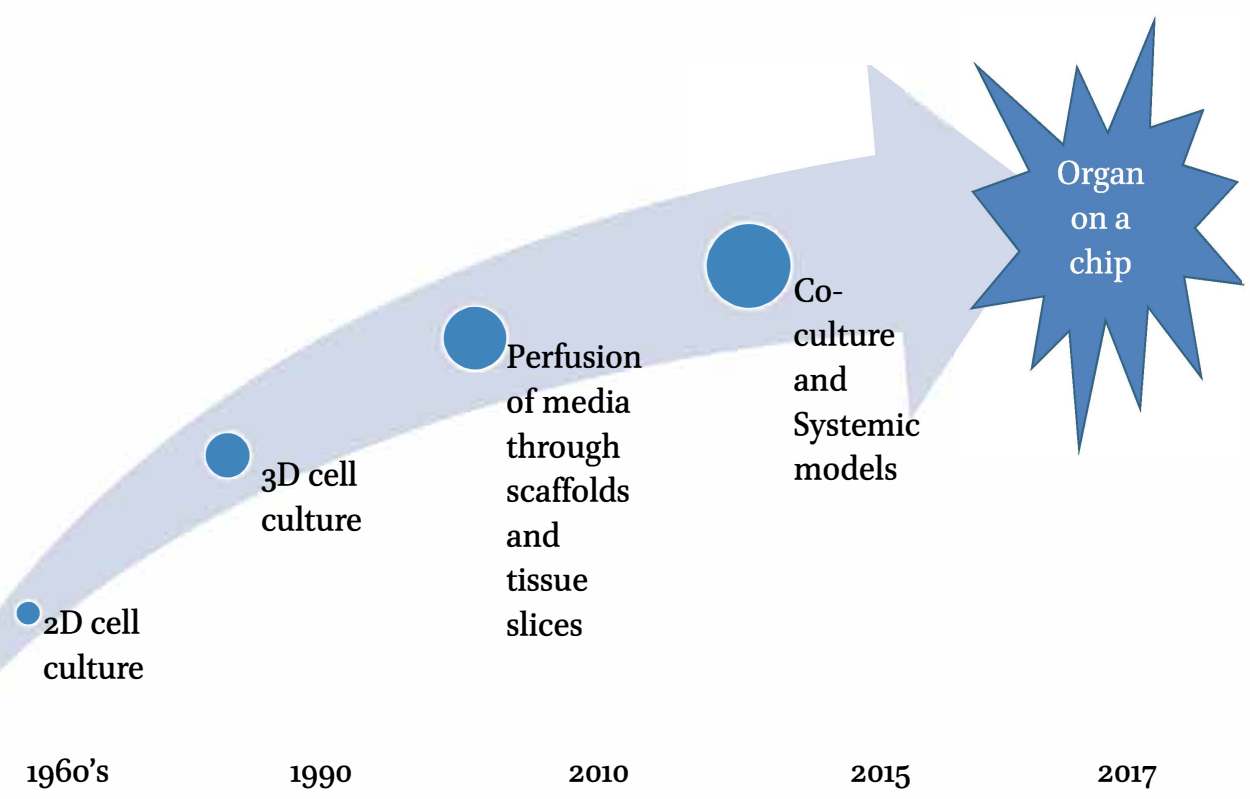

FIGURE 26.1 Developments in in vitro cell culture since the 1960s.

(C) MALCOLM WILKINSON, 2019 | DOI:10.1163/9789004391192_027

This is an open access chapter distributed under the terms of the prevailing cre-By-N fiticemse at the timego-04-39119-2 of publication. 
In this chapter, the following definitions are used:

${ }_{2} D$ cell culture. This is the conventional plating of cells in a plastic or glass plate, such as a petri dish, that contains the liquid culture media. In this format, the cells basal side is in contact with the base wall (glass or plastic); and the top or apical side is in contact with the media. A major limitation of this method is that cell-to-cell contact is very limited. The cell-culture dish can be a classic petri dish or, more likely, one of the highly-miniature versions known as microtiter plates. These have been scaled from 6 wells to $24,96,384$, or even 1,024 , configured as regular arrays in a way that allows robotic equipment to load cells and media and sample media for analysis.

${ }_{3} D$ cell culture. Having recognized the limitation of $2 \mathrm{D}$ cell culture, many approaches have been developed to increase the proportion of cell-to-cell contact to create a more physiologically relevant model of the structure of tissue in the human body. Cells can be provided with a soft or rigid matrix or scaffold in which to grow. Many cells have a natural tendency to adhere to the cellculture dish plastic; but if the plastic is coated with a low adhesion surface, then the cells can clump together to form spheroids containing between 1,000 and 10,000 cells. There are now several well-established commercial systems for producing spheroids in microtiter plates. The main limitation with this method is that cells in the center of larger spheroids are starved of oxygen and media and become necrotic.

Perfusion. In the human body the vascular network links organs and transports oxygen and nutrients to the cells. It is also a vital communication highway for metabolic signaling between tissues. A major current goal of advanced in vitro models is to be able to recapitulate the physiological interactions between tissues in the body connected by the bloodstream. This has enormous potential, as it will enable studies on specific two-way or higher-order organto-organ and tissue interactions. Perfusion or flow of media across the cell culture is a first step in realizing this objective. Unfortunately, the flow of media past cells can induce flow stress, if the flow is higher than the cells might experience in the body. Hence, control of flow rate is critical. Optimized flow levels (which vary from tissue to tissue) can remove the necrosis in spheroids of tissue slices and upregulate cell activity back to levels observed in freshly isolated human primary cells (Vinci et al., 2011).

Organoids. In vitro culture of whole human organs is difficult and expensive. An intense research effort is underway to see if the main functions of a whole organ can be replicated by a much smaller number of cells, perhaps as few as 10,00o. When provided with the right physical, biochemical, and other cues, these cells will often spontaneously differentiate into a morphology that can replicate the features of tissue in specific organs. These small samples 
of biological material are known as organoids. One example is hepatocytes, where bile ducts form (Ramachandran et al., 2015).

Organ on a chip. This is a commonly used term in the popular press that would benefit from a clearer definition. The term chip is borrowed from the semiconductor industry and is a shortening of the term microchip, which is a small (typically fingernail-sized) crystal of silicon that contains millions of transistor circuits. The implication is that biological circuits will be capable of similar scaling in complexity. Unfortunately, the laws of physics apply. Biological cells and liquid fluids do not continue to function correctly as the size is reduced. This is in stark contrast to electrons on transistor circuits, which operate faster and use less power as they are scaled to smaller geometries. Another feature of microelectronic chips is that they are manufactured thousands at a time on a large wafer of silicon, which is one reason why the cost per-chip can be very low. In contrast, organs on a chip are currently manufactured singly or in small batches. There is very little standardization in the manufacturing processes used between the different laboratories making them, apart from the widespread use of silicone, a flexible rubber-like material, to mold the small channels. The chips in this case are typically $2 \mathrm{~cm}$ or more per side (i.e., much larger than silicon chips); and many have fluid connections glued in place to allow cell-culture media or test chemicals to be passed over the cells under culture.

Organ on a plate. This is a larger format approach than organ on a chip and typically has multiple cell-culture chambers molded in a plastic plate. The fluid connections between different chambers can be formed as part of the plate or added by connecting flexible tubing, as in the Quasi Vivo Interconnected Cell Culture System (Yoon et al., 2015). The plastic plate is often designed to meet the industry standard format for 6 well or 24 well to facilitate handling by robotic equipment. Such multi-chamber plates can be used to connect different cell types or run multiple replicates in the same flow system. An integrated systemic view can, thus, be constructed piecewise; and, in the Quasi Vivo advanced cell culture flow systems, allometry can be used to set up physiologically-relevant connected culture models of biotransformation, distribution, adsorption, and gas exchange (Haycock, Ahluwalia and Wilkinson, 2014).

Microphysiological system (MPS). This terminology was first used by the Defense Advanced Research Projects Agency (DARPA) in the United States that launched the MPS program of research. MPS research aims to develop a reconfigurable platform that permits simultaneous study of 10 or more in vitro physiological systems, arranged in any sequence. The goal is to design a flexible, user-friendly, and reliable platform that will allow biological components to interact in a physiologically-relevant manner and will sustain the resident 
tissues for up to four weeks. Researchers developing the in vitro mimics aim to demonstrate that the engineered tissues function together to reproduce each of the human physiological systems. As these system mimics are integrated into a platform of increasing complexity, researchers must demonstrate that the platform reproduces the physiologically-relevant crosstalk between the systems that normally occurs in humans. To validate its behavior, and its potential value for evaluating drugs and vaccines, test compounds with known effects in humans will be applied to the platform. The effects that the test compounds have on the physiological system mimics will then be extrapolated to humans via computer modeling and compared to the health effects previously observed in humans. Many of the current organ on a chip developments are targeted to meet the requirements of the DARPA program.

Although technological capability has now reached the point where multiple cell types can be cultured together in a single miniature plate, it is important to recognize that routine testing of chemical or drug safety lags behind the state of the art by many years. It is only now, after 10 years in development, that ${ }_{3} \mathrm{D}$ cell culture, in the form of spheroids, is being used routinely by industry. However, $2 \mathrm{D}$ cell culture, in 96 or 384 well plates, is still the dominant technology. Considerable evidence of the benefits of any new technique has to be accumulated before industry will adopt it, and it takes even longer before regulatory bodies accept new methods.

One of the driving forces behind the development of the microtiter plate, with 96,384 , or even 1,536 small cavities for cell culture, has been the need to screen very large numbers of chemical compounds for safety or, in the case of the pharmaceutical industry, for their potential as drugs. Historically, the pharmaceutical industry has had access to tens of thousands of chemical compounds that may, potentially, be active drugs. Hence, the need for a very simple go/no go test that could quickly screen a large number of compounds to a more manageable number. Despite the high throughput of tests using microtiter plates, the High Throughput Screening (HTS) assays give very little, if any, information about mechanisms of action. Current HTS assays are very narrowly focused (e.g., receptor binding) and usually use animal-derived cells or subcellular components. If advanced HTS could use human cells/components, they may reveal much more information. Because HTS in vitro testing has only provided poor prediction of what is likely to happen in clinical trials, regulatory bodies still demand testing of drug candidates on animals. However, because 


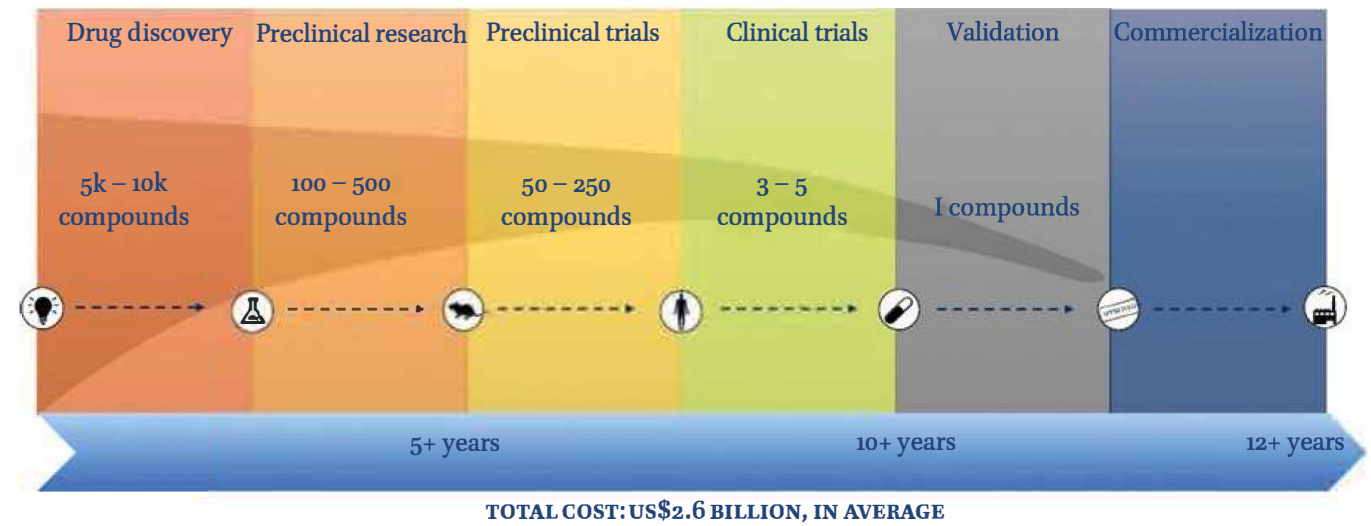

FIGURE 26.2 Summary of the drug development process.

CLERK AND VILLIEN, 2017, REPRINTED WITH PERMISSION

testing on animals is so expensive, pharmaceutical companies try to screen out the more obvious failures before that stage. The drug development process is shown in Figure 26.2. HTS is mainly used in the first stage of drug discovery when the number of candidate drugs to be screened may be as high as 10,000.

There is still considerable opportunity to improve the efficiency of this process despite the billions of dollars invested in it each year. The primary motivation for industry is the immense waste of resources, because around $90 \%$ of the drug candidates that enter clinical trials fail to reach the market (Mullard, 2016). This is because both current in vitro testing and animal methods are poor predictors of what will happen in clinical trials. It is this need for a more predictive method that is driving the development of organ on a chip and organ on a plate technology.

\section{The Functional Requirement for Improved in vitro Methods}

There is a growing body of knowledge that shows that the use of animal cells in in vitro testing is a factor in the poor performance of current methods. Even the use of whole animal models does not replicate what happens in the human body, so it is hardly surprising that animal cells placed in an in vitro environment will also give misleading results (Chandrasekera and Pippin, 2014; Cook, Clerk and Sugden, 2009; Mestas and Hughes, 2004; Potashkin, Blume and Runkle, 2011).

The choice to use animal cells is often driven by convenience rather than scientific reasons for further discussion see Redmond, 2019, Chapter 27. Human cells are difficult to obtain, often come from a single diseased patient, and are not representative of a larger pool of donors. Cell lines derived from 
human cells are more readily available, but there are issues with the cell lineage. Tumor-derived cell lines readily proliferate; but their functionality may be quite different from healthy tissue; and their very robustness may be a problem, if we are trying to achieve a sensitive test for toxicity of a chemical or drug. Even when a representative supply of cells has been secured, there are other reasons why the models can be inadequate. Current research indicates that $2 \mathrm{D}$ static cell culture (no flow of media) is not as good at predicting toxicity as ${ }_{3} \mathrm{D}$ cell culture (Eglen, 2017). There is also a growing body of evidence that perfusion (flow) of media over or through the cells produces a better prediction of IC 50 (half maximal inhibitory concentration) levels of drug toxicity than static medium (Davidge and Bishop, 2017).

Using this wealth of research, we can set out a list of requirements that should be met by any advanced in vitro method, including organ on a chip:

Biological requirements

- Use of human cells

- Culture conditions that produce physiologically relevant organoid models

- Connected organoids, so that the system models the whole organism

- Long-term culture/homeostasis for repeat-dose testing or low clearance compounds

Practical requirements

- Easy to use and fast to set up in the laboratory

- Robust/repeatable across multiple laboratories

- No air bubbles disrupting flow or blockages caused by biological material

Scale requirements

- Better HTS tests and high-content methods to replace animals

- Ability to test thousands of compounds for improving HTS assays

- Ability to test tens of compounds and replace hundreds of animals used in preclinical screening

Economical requirements

- Capital and consumable cost lower than animal testing

- Even lower cost per test to replace HTs.

The last points in this list suggest that the market for advanced cell culture could become segmented into high throughput approaches and high-content (lower throughput) methods.

\section{$4 \quad$ History and Current Status of Organ on a Chip Methods}

The concept of combining of microtechnology and biology to reconstitute the physiological and mechanical functions of human organs is not a new one. 
Michael Shuler at Cornell University was one of its pioneers. In 1994, Shuler filed patents on a macro scale in vitro system for the physiological and metabolic evaluation of substances for use in living beings. The system included one or more cell culture chambers, each containing cells in a culture medium and a gas-liquid exchange device for contacting the culture medium with oxygencontaining gas. By 2003, Shuler was developing a micro-scale version (Park and Shuler, 2003), which was closely followed by Arti Ahluwalia at the University of Pisa (Ahluwalia, 2004), who filed her first patent in 2004. These pioneers were then followed by a surge of researchers, including Luke Lee at the University of California, Linda Griffith at the Massachusetts Institute of Technology, Donald Ingber at Harvard University, Shuichi Takayama at the University of Michigan, and many more. Sung and Shuler (2010) provide an excellent review of this area of research.

Progress between 2004 and 2014 was slow because of the significant technical challenges encountered in the application of microfluidics to biological systems. Few of the start-up companies that emerged from the early academic laboratories managed to get products to market. Despite these early setbacks there has been a resurgence of interest in the area following significant funding allocated to organ on a chip developers by the US DARPA and the US National Institutes of Health $(\mathrm{NIH})$, who have awarded Us $\$ 140$ million and US $\$ 76$ million, respectively, over a 5-year period, to support developments. In parallel, technology developers have raised more than us $\$ 80$ million since 2012 with equity investors. These investments caught the attention of market analysts; and in 2016, the first market forecast specifically aimed at this sector was published (Accuray Research LLP, 2016). In 2017, a more detailed analysis of the market was provided by Yole Research (Clerk and Villien, 2017). Yole's analysts estimated the combined sales of organ on a chip devices and service offerings at no more than US $\$ 7.5$ million in 2016.

Most companies in this area are spin offs from university laboratories and are currently developing their organ on a chip devices through projects sponsored by industrial players. Pharmaceutical and cosmetics companies are interested in the emerging technology but remain skeptical about its potential in the short term. Given the experience with ${ }_{3} \mathrm{D}$ spheroid technology, it could be many years before the technology is ready for widespread adoption. However, the belief that such technologies could become a multibillion dollar market in the mid- to long-term future has the potential to accelerate progress, given the billions of dollars they could help the pharmaceutical industry save every year. Ethical concerns are also one of the potential drivers at the heart of this new market. Around the world, at least 115 million vertebrates are estimated to be used for scientific purposes annually (Taylor et al., 2008). Many of these 


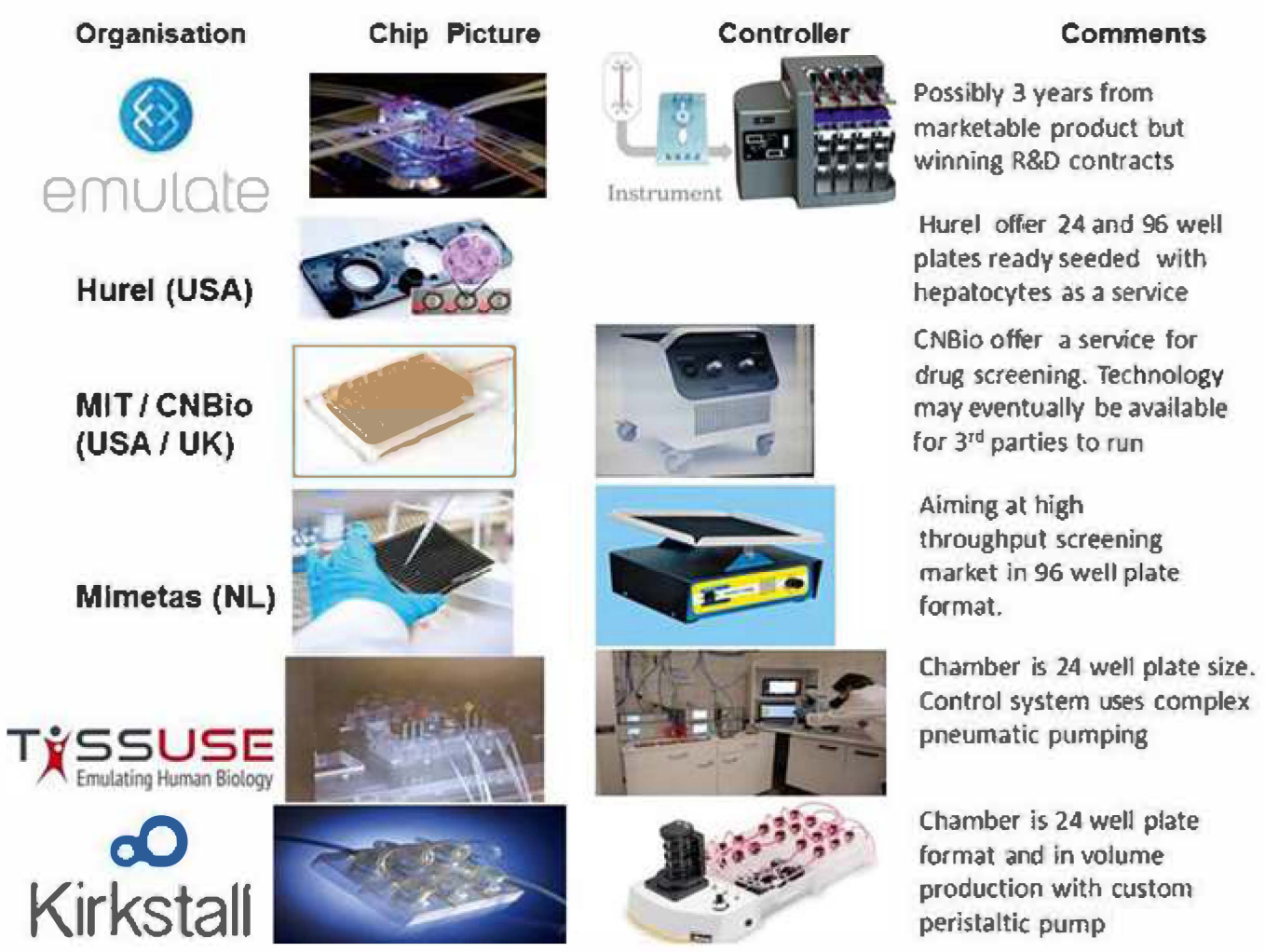

FIGURE 26.3 Leading contenders in the race to develop organs on a chip and organs on a plate.

animals could be replaced by alternative methods, which may include some elements of microfluidic technology.

Some of the leading developments in organ on a chip are summarized in Figure 26.3.

Summarizing the current developments, we can observe that there is little standardization. Each team is developing its own approach, with its own unique technology. The players are mainly start-up companies commercializing prototypes developed in the local universities. There is widespread use of silicone plastic to fabricate chambers and channels; but Mimetas and Kirkstall have opted to use acrylic-type plastic that can be injection molded and, hence, is amenable to volume production. There are widely differing chamber and plate sizes. Hurel, CN Bio, and Mimetas use 96 well plate size; TissUse and Kirkstall use 24 well plate size; and Wyss/Emulate has custom plates for each organ. Another point of divergence is the way that cell-culture media is fed into the chambers and over the cells. Pneumatic, peristaltic, and syringe pumps, as well as gravity fed flow, are all in use. Although some of the cellculture chambers look simple, many require complex control systems to set up and maintain the temperature and gas ambient environment. Mimetas, 
TissUse, and Kirkstall have all opted to use conventional cell-culture incubators into which their systems are loaded.

Returning to the functional requirements listed in the previous section, we can gage how well each of the contenders are faring in their endeavor to achieve a technology that will be capable of replacing some of the animal testing in the drug discovery process.

\subsection{Biological Requirements}

Animal cells may be easier to obtain and keep alive than human primary cells, but they are not moving us forward. Human tumor-derived cell lines are easy to culture but are not representative of healthy tissue. Human-induced pluripotent stem cells (hiPSCs) look promising but are currently expensive and need long, complex protocols to create the differentiated cells needed for organ models. Human donor tissue could be considered the gold standard, but cryopreservation is needed to store tissue from donors to match the time window for experiments. Unfortunately, cryopreservation compromises the function of the cells. Esch, Bahinski and Huh (2015) provide a review of the cell types used in organ on chip models.

\subsection{Practical Requirements}

In order for any new technology to achieve regulatory acceptance, it must demonstrate that it is a robust and repeatable method. Many of the organ on a chip methods are a long way from this goal. They are so complex to set up and operate that they are only running in the host developer's laboratory and offered as a service. In contrast, Kirkstall has designed its Quasi Vivo organ on a plate platform to be easy to use and fast to set up in the laboratory. It is well on the way to demonstrating that it can be robust and repeatable across multiple laboratories with a current academic-user base of more than 70 universities.

\subsection{Scale Requirements}

Figure 26.2 shows the different points in the drug discovery and development process where organs on a chip could be used. There is a clear divergence between the requirement to screen thousands of compounds and improve HTS assays, and the lower throughput needs to test in-depth tens of compounds and replace hundreds of animals used in preclinical screening. Most of the current organ on chip developments have indicated that the former is their commercial goal. In contrast, TissUse and Kirkstall have opted for 24 well plate size chambers that should be more suited to the latter and a focus on animal replacement. 


\subsection{Economical Requirements}

Since so few of the organ on a chip projects have offered products to the market as yet, it is difficult to assess the likely costs involved. Many of the technologies are suitable for scaling to volume production and so, in theory, should be capable of meeting customers' expectations on cost. The economics of animal replacement have been thoroughly researched by Hartung and his team at Johns Hopkins University (Bottini and Hartung, 2009). The cost targets (capital and consumable cost) to replace animal testing are probably easier to meet than those to replace existing HTS.

\section{$5 \quad$ Barriers and Drivers for Change}

There is a clear market need for improvements in the drug discovery and development process, and this is validated by the eagerness with which pharmaceutical and cosmetics companies are evaluating new technologies, one of which is organ on a chip and in silico modeling another. However, inertia among researchers is recognized as a barrier to moving away from existing animal methods (Innovate UK, 2015). In addition, they are conservative and will need time to complete evaluation, validation, and adoption of the technology. Regulatory approval, if required, will take even longer. Apart from the technical challenges yet to be solved, the start-up companies that are championing the new methods face commercial issues. Some have raised significant equity investment to complement government grants and customer-sponsored research and development projects. Government grants to individual companies and to organizations supporting the ${ }_{3}$ Rs have been particularly helpful. In the United Kingdom, such grants have been part of the Innovate UK's roadmap to support the development of non-animal technologies (Innovate $\mathrm{UK}$, 2015).

The current worldwide market for animal testing is estimated to be in excess of us \$30 billion (Bottini and Hartung, 2010); and it is most likely that the companies involved in that market (including contract research organizations offering animal testing) will fight hard to defend their current business, despite the ethical and scientific pressures for change. It is not only businesses that will fight to defend the status quo. Many academic careers are based on animal models, and it is not easy to make changes late in a career. In contrast, early-career researchers will be highly motivated to learn about new methods, but the peer review system for awarding grants will make it tough for them to get approval for ground-breaking and disruptive ideas. Centers of excellence are emerging to support animal replacement technologies. The Center 
for Alternatives to Animal Testing (CAAT) at Johns Hopkins University in the us was one of the first and now has a satellite at the University of Konstanz in Germany. The UK has the Animal Replacement Centre of Excellence (ARC) at Queen Mary University, London; and Canada has just launched the Canadian Centre for Alternatives to Animal Methods (CCAAM) at the University of Windsor, Ontario. These centers will act as nuclei for further awareness creation, research funding, technology evaluation, and industry support. Their most effective action may be to train a new generation of researchers who are aware of the disruptive technology and are willing to become agents for change.

The conservatism of regulators is often cited as one of the most difficult barriers to overcome (Innovate UK, 2015). The production of an overwhelming body of scientific evidence may be the best long-term approach. After all, the regulators' role is to protect the public from the risks of exposure to harmful drugs and chemicals. In the absence of good in vitro models and data, they will always revert to what their colleagues have done for years before them, i.e., insist on animal testing. In the short term, there are other ways to introduce the new technology that do not need regulatory approval but utilize in vitro tests in parallel to reduce the number of drug candidates before they enter the expensive animal and clinical-testing phases. In vitro methods are then effectively being used to cause compounds to fail early, and the potential economic savings are immense. Additionally, as in vitro assays are implemented, they will be validated by improved success in subsequent clinical trials.

Figure 26.4 shows a representative comparison of the economic benefits of using advanced in vitro testing to reduce the number of drug compounds going forward into animal testing. By testing 25 compounds and eliminating 10 that have shown some adverse activity, only 15 go forward. The potential saving is US $\$ 226$ million, justifying a significant investment in the additional in vitro tests. This approach does not need regulatory approval because animal testing will still be used in the later stages, albeit on a reduced number of compounds. With these potential savings per drug candidate reaching the market, Yole Research's projection that the organ on a chip market could reach between us $\$ 60$ million and us $\$ 176$ million by 2022 does not seem unreasonable.

\section{A Strategy for Accelerating the Paradigm Change Away from Animal Use}

The previous sections described the technology, market opportunity, status of current developments, and barriers and incentives for change. Is there any way that we can accelerate this process and speed the adoption of methods that 
Traditional Developement

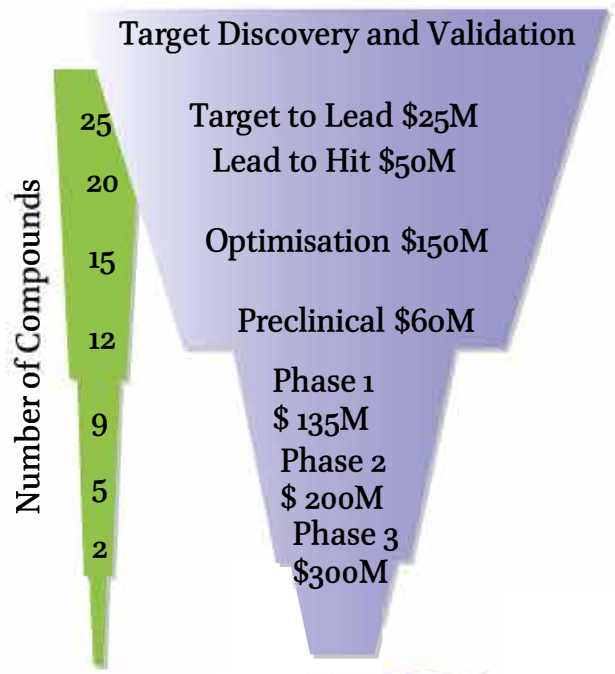

Using Improved in Vitro with OOC

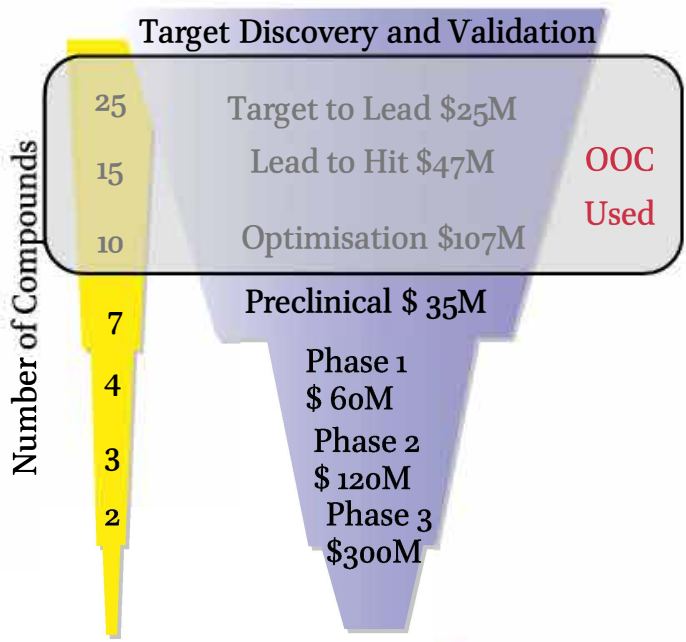

Total cost $\$ 694 \mathrm{M}$

FIGURE 26.4 Potential cost benefits of using advanced in vitro methods, such as organ on a chip (ooc).

will save the lives of countless animals and reduce their suffering? Many years of research in the high technology industry show us that incremental change is much easier to push forward than disruptive change. Clearly, the full replacement of animals is a disruptive change. The candidate technology presented here is also disruptive, as it is not a natural outgrowth of existing biology or microtechnology but a fusion of the two. The technical challenges are enormous and require a multidisciplinary effort from biologists, pharmacologists, statisticians, computer modelers, plastic material and fabrication engineers, and many other experts. It is interesting that the challenge of bringing together scientists from different disciplines to work on, so-called, grand challenges has been addressed in several universities. Pisa University created the Centro Piaggio and Sheffield University the Kroto Centre with this express goal. Technical brilliance is not enough. The new technology has to be translated into a sustainable business, and that takes a whole different skill set. Entrepreneurship is needed.

The roadmap and strategy presented in Figure 26.5 describes an innovative approach to synthesizing disruptive change from a number of smaller, almost incremental steps. The foundation starts with good science from a few opinion 

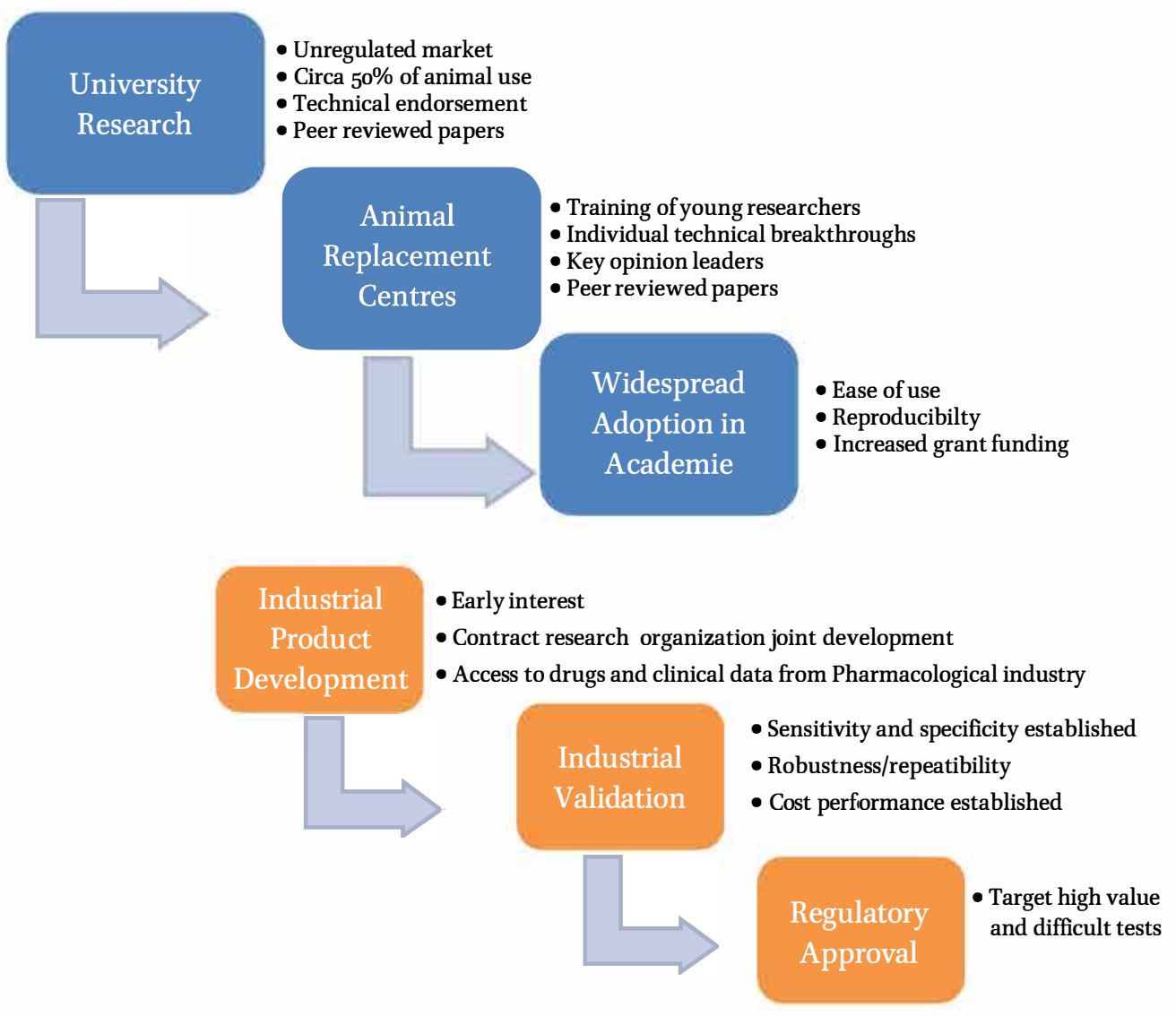

FIGURE 26.5 A roadmap and strategy for accelerating the adoption of alternative methods, showing parallel adoption in academia and industrial research.

leaders in the academic sector but leads on to the creation of centers of excellence that eventually drive widespread adoption of the new paradigm through academe. It is important to note that academic research accounts for about half of the total number of animals used in the UK. Industry adoption follows but is slower at first because of the need for extensive evidence to support claims of superiority for the emerging new technology. The early evidence comes from academics followed by the development of robust protocols by contract research organizations. The pharmacological industry is increasingly using contract research organizations to do validation and development work that may previously have been done in their own research and development laboratories.

Much of the current interest and excitement about organ on a chip technology is fueled by marketing hype and will soon be replaced by disillusionment unless practical working systems are delivered. Many of the venture capitalists investing in organ on a chip will expect immense financial return from the $10 \%$ of their portfolio that succeed. There are some very exciting technology 
developments under way. Although some of these are several years away from the market, there is no doubt that within the next 3 to 5 years, we will see the start of a significant shift away from the use of animals.

\section{References}

Accuray Research LLP (2016). Organ on Chip Market Analysis and Trends - Organ, Application - Forecast to 2025. [online] Available at: https://www.researchandmar kets.com/reports/3951918/organ-on-chip-market-analysis-and-trends-organ [Accessed 12 August 2018].

Ahluwalia, A. (2004). Italian patent application. IT 2004PI00046.

Bottini, A.A. and T. Hartung (2009). Food for Thought.... On the Economics of Animal Testing. Alternatives to Animal Experimentation, 26(1), pp. 3-16.

Chandrasekera, P.C. and J.J. Pippin (2014). Of Rodents and Men: Species-specific Glucose Regulation and Type 2 Diabetes Research. Alternatives to Animal Experimentation, 31(2), pp. 157-176.

Clerk, S. and M. Villien (2017). Organs on Chips 2017, Market and Technology Report. Yole Développement.

Cook, S.A., A. Clerk and P.H. Sugden (2009) Are Transgenic Mice the "Alkahest" to Understanding Myocardial Hypertrophy and Failure.Journal of Molecular and Cellular Cardiology, 46(2), pp. 118-129.

Davidge, K.S. and J. Bishop (2017). Improved IC50 Prediction Using Quasi Vivo ${ }^{\circ}$. Society of Toxicology Annual Meeting, 2017. Baltimore, MD.

Eglen, R. (2017). The Use of Tumour Spheroids in Cancer Research. [video] Available at: https://www.youtube.com/watch?v=pQdiAhNNrfY [Accessed 12 August 2018].

Esch, E.W., A. Bahinski and D. Huh (2015). Organs-on-chips at the frontiers of drug discovery. Nature Reviews Drug Discovery, 14(4), p. 248-260.

Haycock, J., A. Ahluwalia and J.M. Wilkinson (2014). Cellular In Vitro Testing: Methods and Protocols. Singapore: Pan Stanford Publishing, pp. 1-13.

Innovate UK (2015). A non-animal technologies roadmap for the UK. Advancing predictive biology. [online] Available at: https://www.nçrs.org.uk/sites/default/files/ documents/NonAnimalTechCO082_RYE_4_nrfinal2.pdf [Accessed 12 August 2018].

Mestas, J. and C.C.W. Hughes (2004). Of Mice and Not Men: Differences Between Mouse and Human Immunology.Journal of Immunology, 172(5), pp. 2731-2738.

Mullard, A. (2016). Parsing Clinical Success Rates. Nature Reviews Drug Discovery, 15, p. 447.

Park, T.H. and M.Shuler (2003). Integration of Cell Culture and Microfabrication Technology. Biotechnology Progress, 19, pp. 243-253. 
Potashkin, J.A., S.R. Blume and N.K. Runkle (2011). Limitations of Animal Models of Parkinson's Disease. Parkinson's Disease, pp. 1-7.

Ramachandran, S.D., K. Schirmer, B. Münst, S. Heinz, S. Ghafoory, S. Wölfl, K. SimonKeller, A. Marx, C.I. Øie, M.P. Ebert and H. Walles (2015). In Vitro Generation of Functional Liver Organoid-like Structures Using Adult Human Cells. PloS One, 10(10), p. e0139345. [online] Available at: http://journals.plos.org/plosone/article?id=10.1371/ journal.pone.0139345 [Accessed 12 August 2018].

Redmond, C. (2019). When is an alternative not an alternative? Supporting progress for absolute replacement of animals in science. In: K. Herrmann and K. Jayne, eds., Animal Experimentation: Working Towards a Paradigm Change, Vol. 22. Leiden: Brill, pp. 654-672.

Sung, J.H. and M.L. Shuler (2010). In Vitro Microscale Systems for Systemic Drug Toxicity Study. Bioprocess Biosystem Engineering, 33, pp. 5-19.

Taylor, K., N. Gordon, G. Langley, and W. Higgins (2008). Estimates for Worldwide Laboratory Animal Use in 2005. Alternatives to Laboratory Animals, 36, pp. 327-342.

Vinci, B., C. Duret, S. Klieber, S. Gerbal-Chaloin, A. Sa-Cunha, S. Laporte, B. Suc, P. Maurel, A. Ahluwalia and M. Daujat-Chavanieu (2011). Modular Bioreactor for Primary Human Hepatocyte Culture: Medium Flow Stimulates Expression and Activity of Detoxification Genes. Biotechnology Journal, 6(5), pp. 554-564.

Yoon, M., J. Pedersen, Y. Zhao and H.J. Clewell (2015). Why and How Do We Incorporate Metabolism and Kinetics in Integrated Testing and Assessment. 4th Annual ASCCT Meeting. Durham, NC. 